\title{
Multiple Signature Processing-Based Fault Detection Schemes for Broken Rotor Bar in Induction Motors
}

\author{
Bulent Ayhan, Student Member, IEEE, Mo-Yuen Chow, Senior Member, IEEE, and Myung-Hyun Song, Member, IEEE
}

\begin{abstract}
The existence of broken rotor bars in induction motors can be detected by monitoring any abnormality of the spectrum amplitudes at certain frequencies in the motor current spectrum. It has been shown that these broken rotor bar-specific frequencies are settled around the fundamental stator current frequency and are termed lower and upper sideband components. Broken rotor bar fault detection schemes should depend on multiple signatures in order to overcome or reduce the effect of any misinterpretation of the signatures that are obscured by factors such as measurement noises and different load conditions. Multiple discriminant analysis (MDA) provides an appropriate environment to develop such fault detection schemes because of its multi-input processing capabilities. The focus of this paper is to provide a new fault detection methodology for broken rotor bar fault detection and diagnostics in terms of its multiple signature processing feature and the motor operation partitioning concept to improve the overall detection performance. This paper describes two fault detection schemes within this methodology, and demonstrates that multiple signature processing is more efficient than single signature processing. The first scheme, which will be named the "monolith scheme," is based on a single large-scale MDA unit representing the complete operating load torque region of the motor, while the second scheme, which will be named the "partition scheme," consists of many small-scale MDA units, each unit representing a particular load torque operating region.
\end{abstract}

Index Terms-Broken rotor bar, discriminant analysis, fault diagnosis, induction motors, monitoring.

\section{INTRODUCTION}

$\mathbf{I}$ NDUCTION motors have dominated the field of electromechanical energy conversion, featuring $80 \%$ of the motors in use [1]. The applications of induction motors are widespread. Some induction motors are key elements in assuring the continuity of the process and production chains of many industries. A majority of induction motors are used in electric utility industries, mining industries, petrochemical industries, and domestic appliances industries. The list of the industries and applications that induction motors take place in is rather long. Induction motors are also often used in critical applications such as nuclear plants, aerospace, and military applications, where the reliability must be of high standards.

Induction motors often operate in hostile environments such as corrosive and dusty places. They are also exposed to a variety

Manuscript received September 3, 2003; revised December 2, 2003. This work was supported in part by the National Science Foundation under Grant ECS-0245383. Paper no. TEC-00229-2003.

B. Ayhan and M.-Y. Chow are with the Department of Electrical and Computer Engineering, North Carolina State University, Raleigh, NC 27695 USA (e-mail: bayhan@unity.ncsu.edu; chow@eos.ncsu.edu).

M.-H. Song is with the Department of Electrical Control Engineering, Sunchon University, Maegokdong 315, Korea (e-mail: mhsong@sunchon.ac.kr).

Digital Object Identifier 10.1109/TEC.2004.842393 of undesirable conditions and situations such as misoperations. These unwanted conditions can cause the induction motor to go into a premature failure period, which may result in an unserviceable condition of the motor, if not detected at its early stages of the failure period [2], [3]. The early detection of the incipient motor fault is thus of great concern. Rotor failures are among these failures, and they now account for $5 \%-10 \%$ of total induction motor failures [4]. Several monitoring techniques have been developed for broken rotor bar fault detection, most of which are based on motor current signature analysis (MCSA). In recent years, several advanced signal processing techniques such as high-resolution spectral analysis, higher-order statistics, and wavelet analysis have been applied to broken rotor bar and other motor fault detection problems [1].

MCSA techniques include parametric and nonparametric spectrum analysis methods of motor current in general. In the parametric methods, autoregressive models have been fitted with time series of the signal, and model parameters have been used in order to compute the frequency spectrum. Nonparametric methods, on the other hand, are based on Fourier transform and search for periodicities of the signal. Power spectral density (PSD) analysis of motor current is one of the widely used MCSA techniques. There are several approaches to calculate PSD. The periodogram method and Welch's periodogram methods are two of the nonparametric spectrum methods.

Broken rotor bar fault in induction motors can be detected by monitoring any abnormality of the motor current power spectrum amplitudes at several certain frequency components. These frequency components are located around the main frequency line and are determined according to the number of poles and mechanical speed of the motor. However, there are other effects that may obscure the detection of the broken rotor bar fault or cause false alarms. For example, these effects can be intrinsic manufacturing dissymmetry, or load torque oscillation that can produce stator currents with the frequency values that are the same as the monitored frequencies. In monitoring these frequency components, it is assumed that the load torque is constant. Any variation of the load torque with the rotational speed can produce frequency harmonics, which may overlap the harmonics caused by broken rotor bar fault [5]. A broken rotor bar fault detection scheme based on multiple frequency signatures thus should be more reliable in overcoming or reducing the effect of misinterpreted signatures, which are caused by the effects discussed formerly or some other unknown reasons. Multiple discriminant analysis (MDA) provides an appropriate environment to develop such fault detection schemes because of its multi-input processing capabilities. 
The focus of this paper is to provide a new fault detection methodology for broken rotor bar fault detection and diagnostics in terms of its multi signature processing feature and the motor operation partitioning concept to improve the overall detection performance. This paper presents two fault detection schemes within this methodology, and demonstrates that multiple signature processing is more effective than single signature processing. The first scheme will be named the "monolith scheme," and is based on a single MDA unit representing the complete motor operating load torque region. The second scheme will be named the "partition scheme," and it consists of several small MDA units, each of which represents a particular load torque operating region. In the partition scheme, the computational load and complexity of the single units in the monolith scheme are distributed to smaller units, which, as a result, increase the broken rotor bar fault detection performance. The partition scheme thus can be thought of as partitioning a highly nonlinear mapping problem in a high dimension space into linear or low-order nonlinear mapping problems in a reduced dimensional space. The two detection schemes have been investigated using experimental data.

This paper is organized as follows: Section II discusses the frequencies of interest to the broken rotor bar problem and outlines the frequencies to be used in the MDA units. Section III briefly describes the fundamental properties of MDA. Section IV presents the experiment setup and motor data specifications. Section V outlines the fault detection schemes together with experimental results and analysis. Section VI concludes the findings of this paper.

\section{Motor CURRENT SPECTRAL COMPONENTS FOR BROKEN ROTOR BAR}

Kliman and Filipetti [6], [7] used MCSA methods to detect broken rotor bar faults by investigating the sideband components around the supplied current fundamental frequency (i.e., the line frequency) $f_{o}$

$$
f_{b}=(1 \pm 2 s) f_{o}
$$

where $f_{b}$ are the sideband frequencies associated with the broken rotor bar, and $s$ is the per-unit motor slip. The slip $s$ is defined as the relative mechanical speed of the motor $n_{m}$ with respect to the motor synchronous speed $n_{s}$ as

$$
s=\frac{n_{s}-n_{m}}{n_{s}} .
$$

The motor synchronous speed $n_{s}$ is related to the line frequency $f_{o}$ as

$$
n_{s}=\frac{120 f_{o}}{P}
$$

where $P$ is the number of poles of the motor and the constant " 120 " is used to express the motor synchronous speed $n_{s}$ in revolutions per minute ( $\mathrm{r} / \mathrm{min}$ ) unit.

The broken bars also give rise to a sequence of other sidebands given by [7]

$$
f_{b}=(1 \pm 2 k s) f_{o}, k=1,2, \ldots, k_{n}, \text { where } f_{b}>0 \forall k
$$

and are depicted conceptually in Fig. 1.

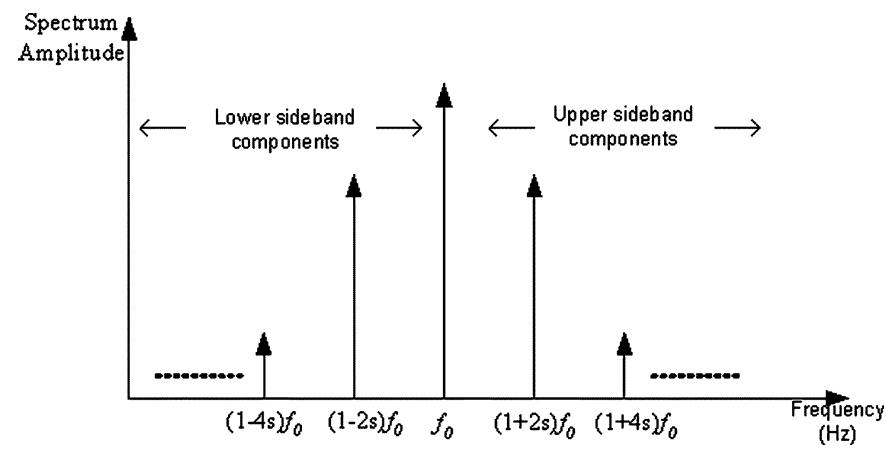

Fig. 1. Sideband frequencies around the fundamental line frequency.

Fig. 1 shows the frequency components specific to a broken rotor bar fault, which is given in (4) for $k=1$ and 2. These frequencies are located around the fundamental line frequency and are termed as lower sideband and upper sideband components as indicated in Fig. 1. There are other spectral components that can be observed in the stator line current due to the broken rotor bar fault [6]

$$
f_{b}=\left[\left(\frac{k}{p}\right)(1-s) \pm s\right] f_{0}, \quad \text { where } \frac{k}{p}=1,3,5, \ldots
$$

The fault detection schemes investigated in this paper depend on multiple signature processing. These signatures correspond to the power spectrum amplitudes of the motor current (phase-a) data at the selected frequencies. A detailed explanation about the experiment setup and specifications of the collected motor data will be given in Section IV. We use Welch's periodogram method to compute the power spectrum of phase-a motor current data. In Welch's periodogram method, we apply a Hanning window and 50\% overlapping percentage among the partitioned segments. It has been noted that the use of a Hanning window and 50\% overlap leads to an efficient implementation of the fast Fourier transform (FFT) algorithm [8]. In the fault detection schemes, we consider four of the broken rotor bar fault-specific frequency components. Let $\mathbf{F}=\left\{f_{1^{-}}, f_{1^{+}}, f_{2^{-}}, f_{2^{+}}\right\}$be the set of broken rotor bar fault-specific frequency components, and $\mathbf{P}=\left\{p_{1^{-}}, p_{1^{+}}, p_{2^{-}}, p_{2^{+}}\right\}$be the set of Welch's spectrum amplitudes at these frequencies. The frequency components in set $\mathbf{F}$ are $f_{1^{-}}=(1-2 s) f_{0}, f_{1^{+}}=(1+2 s) f_{0}$, $f_{2^{-}}=(1-4 s) f_{0}$, and $f_{2^{+}}=(1+4 s) f_{0}$, where $f_{0}$ is the fundamental stator current frequency and $s$ is the slip. $f_{1^{-}}$and $f_{1^{+}}$are the first lower and upper sidebands, while $f_{2^{-}}$and $f_{2^{+}}$ are the second lower and upper sidebands around $f_{0}$, which are expressed mathematically in (4). The inputs for the MDA units in the monolith and partition schemes thus consist of the signature set $\left\{p_{1^{-}}, p_{1^{+}}, p_{2^{-}}, p_{2^{+}}\right\}$.

The motivation in choosing the first and second lower and upper sidebands around the fundamental supply frequency is due to a higher signal-to-noise ratio (SNR) of these harmonics, which contain more reliable and discriminative information when compared to other harmonics. The higher frequency harmonics are relatively low in spectrum amplitude, they are thus more sensitive to noise. However, there is no restriction in including other signatures or increasing the number of signatures within the investigated detection schemes, as far as the 
included signatures provide discriminative information about the broken rotor bar fault.

The methodology in the investigated fault detection schemes uses the Welch's spectrum amplitudes of motor current data at the broken rotor bar fault-specific frequencies as the discriminative signatures in the fault detection decision. However, the locations of the monitored frequencies $f_{b}$ depend on the slip factor $s$, which is a function of the motor mechanical speed. When the no-load case of the motor is considered, the slip factor value approaches 0 . This results in the interference of the monitored frequencies with the main supply frequency or its harmonics. The current detection schemes will thus not be able to perform efficiently for the no-load condition of the motor. On the other hand, signatures that are not dependent on the slip factor and that carry discriminative information, can be included within the methodology in order to make the detection schemes provide reliable fault decision in the no-load condition of the motor.

\section{MultiPle DiscriminANT ANALYSIS}

In this section, we will briefly describe the fundamental properties of MDA, which is used as the detection methodology in the fault detection schemes.

Multiple discriminant analysis considers the problem of assigning individuals to certain groups that have been identified beforehand. The objective in multiple discriminant analysis is thus to find a set of linear combinations of the variables whose values are as close as possible within groups and as far apart as possible between groups [9]. Let $\mathbf{X}=\left[x_{i j}\right]$ be the data matrix with $m$ rows and $n$ columns, where each row stands for the observations and each column stands for the variables. The mean of the $j^{\text {th }}$ column variable in $\mathbf{X}, \bar{x}_{j}$, can then be expressed as

$$
\bar{x}_{j}=\frac{1}{m} \sum_{i=1}^{m} x_{i j} .
$$

Suppose that the $m$ rows of $\mathbf{X}$ are separated into $q$ groups, and each group is defined by a set $I_{k}$ of $m_{k}$ values of index $i$, where the relation between $m_{k}$ and $m$ is expressed in (7)

$$
\sum_{k=1}^{q} m_{k}=m
$$

Let $\bar{x}_{k j}$ be the mean of the $j^{\text {th }}$ column variable in group $k$; $\bar{x}_{k j}$ can then be expressed as

$$
\bar{x}_{k j}=\frac{1}{m_{k}} \sum_{i \in I_{k}} x_{i j} .
$$

We can also express the mean $\bar{x}_{j}$ as

$$
\bar{x}_{j}=\sum_{k=1}^{q} \frac{m_{k}}{m} \bar{x}_{k j}
$$

The total covariance between two variables can be described in two different ways, which are expressed in (10) and (11), respectively

$$
\begin{aligned}
& \operatorname{cov}\left(j, j^{\prime}\right)=\frac{1}{m} \sum_{i=1}^{m}\left(x_{i j}-\bar{x}_{j}\right)\left(x_{i j^{\prime}}-\bar{x}_{j^{\prime}}\right) \\
& \operatorname{cov}\left(j, j^{\prime}\right)=\frac{1}{m} \sum_{k=1}^{q}\left[\sum_{i \in I_{k}}\left(x_{i j}-\bar{x}_{j}\right)\left(x_{i j^{\prime}}-\bar{x}_{j^{\prime}}\right)\right] .
\end{aligned}
$$

The total covariance can be decomposed into two parts: one of the two parts stands for the within-group variances and the other part stands for the between-group variances. This decomposition is known as Huyghens decomposition formula [9] and is expressed in (12)

$$
\begin{aligned}
\operatorname{cov}\left(j, j^{\prime}\right)=\frac{1}{m} \sum_{k=1}^{q} & {\left[\sum_{i \in I_{k}}\left(x_{i j}-\bar{x}_{k j}\right)\left(x_{i j^{\prime}}-\bar{x}_{k j^{\prime}}\right)\right] } \\
& +\sum_{k=1}^{q} \frac{m_{k}}{m}\left(x_{k j}-\bar{x}_{j}\right)\left(x_{k j^{\prime}}-\bar{x}_{j^{\prime}}\right) .
\end{aligned}
$$

Equation (12) can be interpreted as the sum of the covariance within the groups (first term) and the covariance between the groups (second term), and is expressed in matrix form as

$$
\mathbf{T}=\mathbf{W}+\mathbf{B}
$$

where $\mathbf{T}$ denotes the total covariance, $\mathbf{W}$ denotes the covariance within the groups, and $\mathbf{B}$ denotes the covariance between the groups.

In order to define the problem addressed by multiple discriminant analysis, let us introduce $a(i)$, where $a(i)$ is equal to the value of a linear combination of the $n$-centered variables for individual $i$

$$
a(i)=\sum_{j=1}^{n} a_{j}\left(x_{i j}-\bar{x}_{j}\right) .
$$

The variance $\operatorname{var}(\mathbf{a})$, where $\mathbf{a}$ is the vector composed of components $a_{1}, \ldots, a_{n}$, can then be expressed as

$$
\operatorname{var}(\mathbf{a})=\frac{1}{m} \sum_{i=1}^{m} a^{2}(i)=\frac{1}{m} \sum_{i=1}^{m}\left[\sum_{j=1}^{n} a_{j}\left(x_{i j}-\bar{x}_{j}\right)\right]^{2} .
$$

By expanding (15), $\operatorname{var}(\mathbf{a})$ is found as

$$
\operatorname{var}(\mathbf{a})=\frac{1}{m} \sum_{i=1}^{m} \sum_{j=1}^{n} \sum_{j^{\prime}=1}^{n} a_{j} a_{j^{\prime}}\left(x_{i j}-\bar{x}_{j}\right) .
$$

Equation (16) can be expressed in matrix form as $\mathbf{a}^{T} \mathbf{T a}$. The variance of a linear combination a of the variables can then be separated into within variance and between variance as $\mathbf{a}^{T} \mathbf{T a}=$ $\mathbf{a}^{T} \mathbf{W a}+\mathbf{a}^{T} \mathbf{B a}$. The objective of multiple discriminant analysis is to find a linear combination of the variables that have a maximum between groups variance and a minimum within groups 


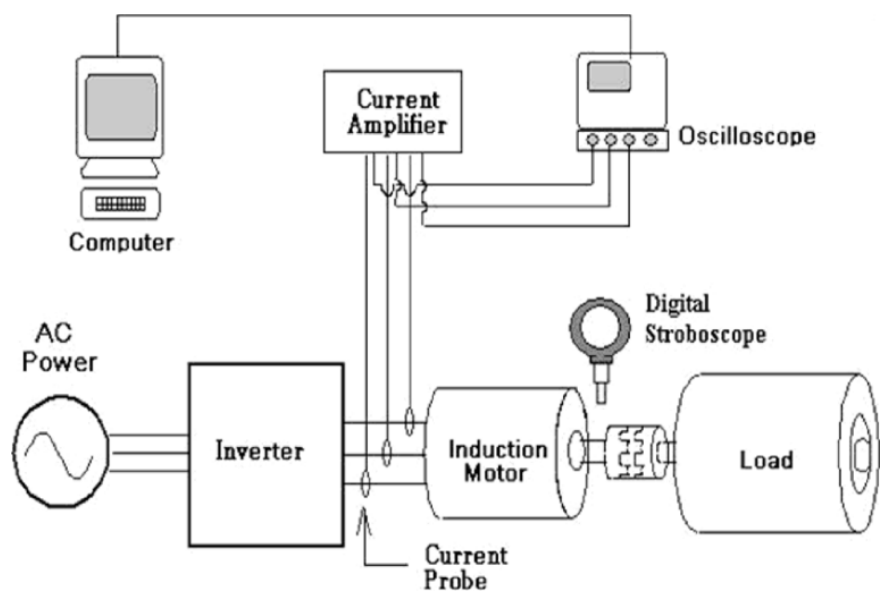

Fig. 2. Motor data collection scheme.

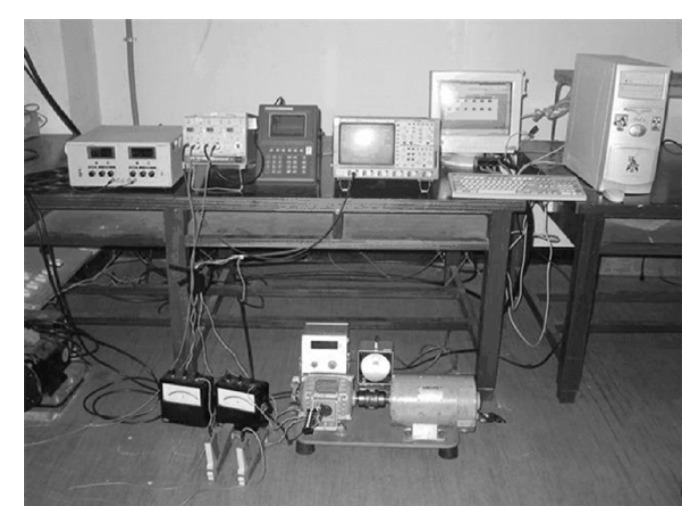

Fig. 3. Actual experiment setup to collect healthy and faulty motor data.

variance. The aim is to find a such that $\mathbf{a}^{T} \mathbf{B a} / \mathbf{a}^{T} \mathbf{W a}$ is $\max -$ imum. This linear combination is termed as the linear discriminant function. The process to find the linear discriminant function is well described in the literature, and readers can refer to [9] for further information.

MDA with linear discriminant functions is perhaps the most widely used discriminant analysis technique and is also known as LDA. In addition, there are many MDA methods developed with nonlinear discriminant functions for problems with nonlinear classification boundaries [9].

\section{EXPERIMENT SETUP AND Motor Data SpeCiFications}

We performed invasive experiments on an actual induction motor for our analyses. The characteristics of the three-phase induction motor used in our experiment are listed in Table I. The motor was tested with a healthy rotor and with a faulty rotor that had one broken rotor bar. The broken rotor bar fault was induced by filling one of the rotor bars with anchoring cement before the diecasting process. Anchoring cement is a high-strength, fastsetting gypsum cement with low conductivity. The overall data collection scheme and the actual experiment setup picture are depicted in Figs. 2 and 3, respectively.

The induction motor was fed through a three-phase ABB, ACS 501 inverter. A Tektronix TM 5003 current amplifier amplifies the induction motor stator currents before they are sent to the interfacing Pentium PC through the oscilloscope. The needed load
TABLE I

INDUCTION MOTOR CHARACTERISTICS USED IN THE EXPERIMENT

\begin{tabular}{l|l}
\hline Description & Value \\
\hline Motor Manufacturer & HYOSUNG, Korea \\
\hline Motor Brand & HSX0504211 \\
\hline Power & $0.75 \mathrm{~kW}(1 \mathrm{Hp})$ \\
\hline Input Voltage & $380 \mathrm{~V}$ \\
\hline Full Load Current & $2.2 \mathrm{~A}$ \\
\hline Supply Frequency & $60 \mathrm{~Hz}$ \\
\hline Number of Poles & 4 \\
\hline Number of Rotor Slots & 44 \\
\hline Number of Stator Slots & 36 \\
\hline Full Load Torque & $0.43 \mathrm{~kg} \cdot \mathrm{m}$ \\
\hline Full Load Speed & $1690 \mathrm{rpm}$ \\
\hline
\end{tabular}

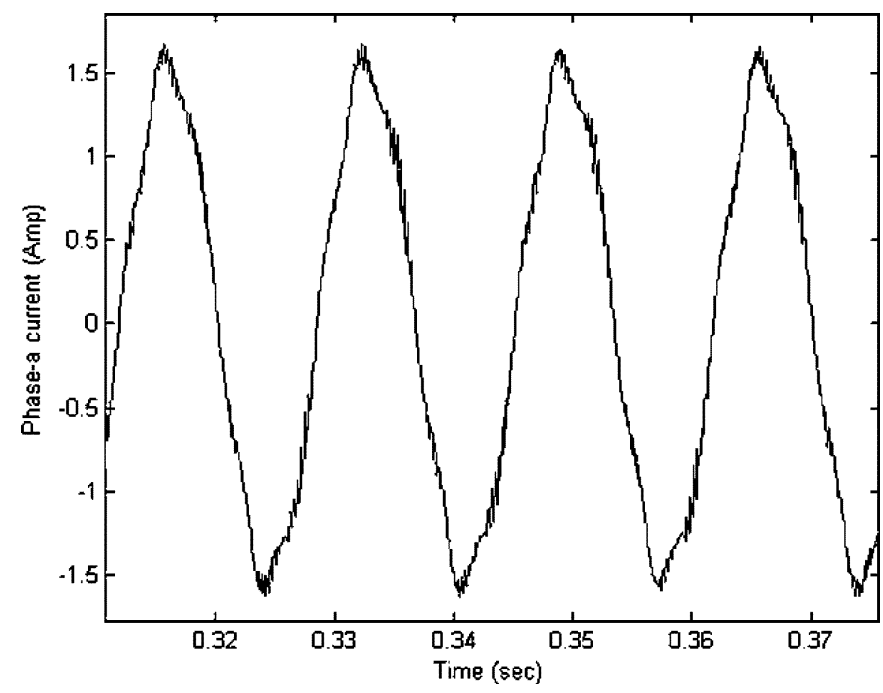

Fig. 4. Healthy phase-a motor current data collected from the experiment setup under full-load condition of the motor.

condition of the induction motor was established by connecting the test motor to a dc motor, which is used as a generator and is capable of simulating any desired load condition. The speed of the induction motor was measured by a digital stroboscope.

The experiments involved collecting phase-a stator induction motor current data for spectrum signature extraction and speed data for computing locations of the broken rotor bar-specific frequencies at four different load conditions of the motor, both with one broken rotor bar fault and without any fault. The load conditions of the motor are $25 \%, 50 \%, 75 \%$, and full load, respectively. These load condition percentages are determined according to the motor nameplate information given in Table I. Thus, there are a total of eight different experiment cases. For each individual case, 20 sets of motor current data were collected with a sampling frequency of $10 \mathrm{kHz}$. Thus, each motor current data set contains 10000 samples for a duration of $1 \mathrm{~s}$. A sample phase-a motor current data waveform corresponding to a healthy state of motor under full-load condition is demonstrated in Fig. 4.

The investigated fault detection schemes require motor current, motor speed data, and motor load condition information, which are easily accessible measurements through the motor. Thus, the investigated fault detection schemes can be used in motor manufacturing facilities, or assembly plants for quality control testing, without disturbing the motors' normal operation. 


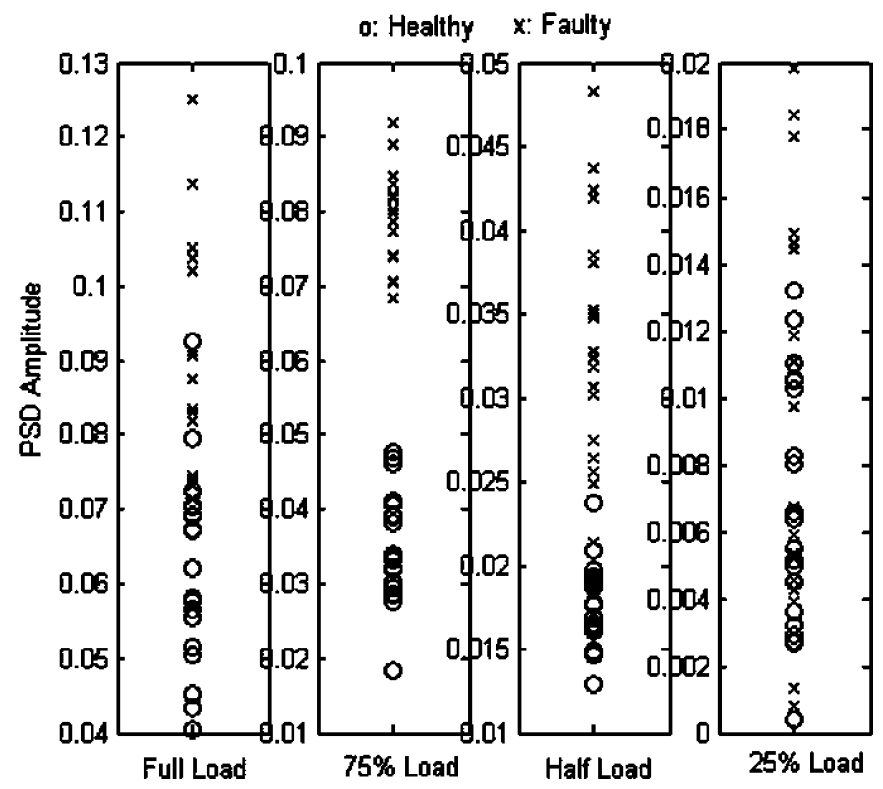

Fig. 5. PSD estimates at $f_{1-}=(1-2 s) f_{o}$ under motor load conditions $T_{L} \in\left\{T_{L_{100 \%}}, T_{L_{75 \%}}, T_{L_{50 \%}}, T_{L_{25 \%}}\right\}$.

\section{FAult Detection Schemes AND ExPerimental Results}

Let $C$ denote the motor condition, which is comprised of the two states of the motor: healthy condition $H$ and faulty condition with one broken rotor bar $F C \in\{H, F\}$. From the motor current power spectrum analysis and broken rotor bar-specific frequency components knowledge, there exists a mapping $\mathfrak{M}$ from $\left(\mathbf{P}, T_{L}\right)$ to $C$ as expressed in (17)

$$
\mathfrak{M}:\left(\mathbf{P}, T_{L}\right) \rightarrow C
$$

where $\mathbf{P}=\left\{p_{1^{-}}, p_{1^{+}}, p_{2^{-}}, p_{2^{+}}\right\}$is the set of Welch's periodogram power spectrum amplitudes at frequencies $\mathbf{F}=\left\{f_{1^{-}}, f_{1^{+}}, f_{2^{-}}, f_{2^{+}}\right\}$and $T_{L}$ is the motor load condition. In our experiments, we have collected data at four different load conditions of the motor as discussed in Section III, where $T_{L}$ is either at full load, $75 \%, 50 \%$, or $25 \%$ load, $T_{L} \in\left\{T_{L_{100 \%}}, T_{L_{75} \%}, T_{L_{50 \%}}, T_{L_{25 \%}}\right\}$. In these experiments, motor speed variation in the collected motor speed data is less than $1.4 \%$ in all of the four investigated motor load conditions. Fig. 5 demonstrates the PSD estimates at one of the broken rotor bar fault-specific frequencies $f_{1^{-}}=(1-2 s) f_{o}$ under four different motor load conditions $T_{L} \in\left\{T_{L_{100 \%}}, T_{L_{75 \%}}, T_{L_{50 \%}}, T_{L_{25 \%}}\right\}$. In this figure, symbol "o" represents the PSD estimate of a healthy motor data, while symbol " $X$ " represents the PSD estimate of a faulty motor data with one broken rotor bar.

In the monolith scheme, we use a single fault mapping unit $\mathfrak{M}$, which maps the signatures extracted throughout the complete motor operating load condition to the motor condition. This scheme is depicted in Fig. 6. Inputs of the MDA mapping unit consist of the signature set $\left\{T_{L}, p_{1^{-}}, p_{1^{+}}, p_{2^{-}}, p_{2^{+}}\right\}$, where $T_{L}$ is the motor load condition, $p_{1^{-}}, p_{1^{+}}$are the first and $p_{2^{-}}, p_{2^{+}}$are the second lower and upper sidebands' Welch's periodogram power spectrum amplitudes around the fundamental

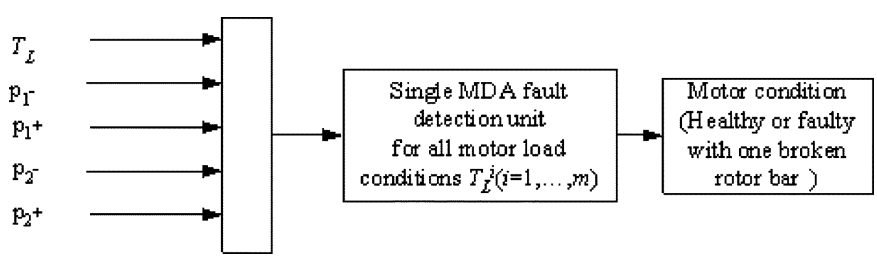

Fig. 6. Monolith scheme.

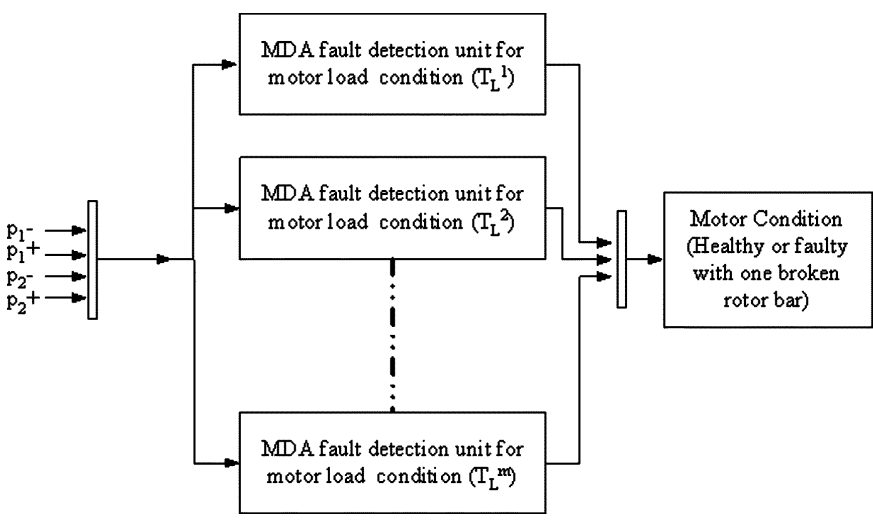

Fig. 7. Partition scheme.

current frequency $f_{o}$, respectively. The mapping for the monolith scheme is depicted in (18)

$$
\mathfrak{M}:\left(p_{1^{-}}, p_{1^{+}}, p_{2^{-}}, p_{2^{+}}, T_{L}\right) \rightarrow\{H, F\} .
$$

In the partition scheme, the single mapping unit $\mathfrak{M}$, which is used in the monolith scheme, is divided into disjoint submapping units $\mathfrak{M}_{i}$, where $\mathfrak{M}_{i}$ corresponds to a submapping unit for a particular motor load condition and, $\mathfrak{M}=\bigcup_{i=1}^{m} \mathfrak{M}_{i}, \bigcap_{i=1}^{m} \mathfrak{M}_{i}=$ $\varnothing$. In our case, $m=4$. Thus, the submapping units in the partition scheme consist of the spectrum amplitude signatures only. A submapping unit is depicted in (19)

$$
\mathfrak{M}_{i}:\left(p_{1^{-}}, p_{1^{+}}, p_{2^{-}}, p_{2^{+}}\right) \rightarrow\{H, F\} .
$$

The conceptual diagram of the partition scheme is presented in Fig. 7. The mapping units in the partition scheme provide partitioning of the complete motor operating load region into subregions, each subregion corresponds to a constant load condition. This procedure thus transforms the nonlinear mapping problem into linear mapping problems or mapping problems with a lower order of nonlinearities. The partition scheme needs motor load condition information as a prerequisite for the preparation of the corresponding mapping units.

In our case, we partition the motor's load operating region into four subregions, depicted as $T_{L}=\left\{T_{L_{100 \%}}, T_{L_{75 \%}}, T_{L_{50 \%}}, T_{L_{25} \%}\right\}$. We then form MDA units for each particular load subregion using the corresponding motor current signatures.

For analyses and performance comparisons of the fault detection schemes with MDA units, two different cases are considered in this paper.

Case 1: Motor signature data set is treated as one whole training data set, where DataSet $\mathrm{D}=$ $\{$ Training Set $\}=\{$ Test Set $\}$. 
Case 2: Motor signature data set is separated into two sets: training and test, where DataSet $\mathbf{D}=$ $\{$ Training Set $\} \cup\{$ Test Set $\}$ and $\{$ Training Set $\} \cap$ $\{$ Test Set $\}=\varnothing$.

In this work, experimental motor data are collected such that they fall into two main groups: healthy state and faulty state of the motor (with one broken rotor bar). We have applied linear discriminant functions in the MDA units depicted in the monolith and the partition schemes for both Case 1 and Case 2; thus, we will use the term LDA instead of MDA in the remainder of this section and the following section of this paper. The training process for the two cases consists of computing the coefficients of the linear discriminant functions. Detailed information about the general principles of MDA with the emphasis on linear discriminant functions is presented in Section III.

In order to compare the fault detection performances of single signature and multiple signature processing, we have applied LDA to each of the four signatures individually for both Case 1 and Case 2.

In analyzing fault detection performances of the two detection schemes, we will use statistical hypothesis testing, Type I error $\alpha$, and Type II error $\beta$. Hypothesis testing is one of the most common aspects in statistical inference. An experimenter first determines a hypothesis about a population parameter. This hypothesis is termed as "null hypothesis" and is depicted with the notation $H_{0}$. The term "population" is used to indicate that the population is composed of an entire set of objects or observations that have something in common. In our case, for example, healthy and faulty motor data are two different populations. The aim of hypothesis testing is to test the applicability of the null hypothesis in the knowledge of experimental data. If the null hypothesis is rejected while it is actually true, this error is termed as Type I error $\alpha$. In a similar way, if the null hypothesis fails to be rejected while it is actually false, this error is termed as Type II error $\beta$. The definitions of Type I and Type II error are depicted in Table II. We use a hypothesis test to analyze the fault detection performances of the investigated monolith and partition schemes with LDA. The null hypothesis $H_{0}$ in our case, is stated as

$H_{0} \quad$ incoming motor signature test data corresponding to the healthy state of the motor.

Type I error $\alpha$ will then correspond to the ratio of the healthy motor data, which are classified as faulty according to LDA, to the total number of motor data. Likewise, Type II error $\beta$ will correspond to the ratio of the faulty motor data, which are classified as healthy, to the total number of motor data. Apparently, Type II error has more severe consequences than Type I error in engineering applications. For example, in a motor manufacturing company, Type II error causes labeling faulty motors as healthy motors and shipment of these faulty motors to the consumers. With Type I error, on the other hand, healthy motors are labeled as faulty; however, a further analysis of these motors helps to identify and fix the problem without affecting any consumers.

We will use the term "correct detection rate," (CDR), in our analyses, which is mathematically expressed in (20)

$$
\mathrm{CDR}=(1-\alpha-\beta) .
$$

Tables III and IV depict the CDRs of each single signature under Cases 1 and 2 together with Type I-II error measures.
TABLE II

TYPE I AND TYPE II ERROR DEFINITION

\begin{tabular}{l|l|l}
\hline Decision & $H_{0}$ is true & $H_{0}$ is false \\
\hline Reject $H_{0}$ & Type I error & Correct \\
\hline Do not reject $H_{0}$ & Correct & Type II error \\
\hline
\end{tabular}

TABLE III

CDRS OF SingLE-FREQUENCY COMPONENTS UNDER CASE 1

\begin{tabular}{l|r|r|r|r}
\hline & \multicolumn{4}{|c}{ Correct Detection Rate (CDR) } \\
\hline & $(1-2 s) f_{o}$ & $(1+2 s) f_{o}$ & $(1-4 s) f_{o}$ & $(1+4 s) f_{o}$ \\
\hline CDR & $132 / 160$ & $150 / 160$ & $152 / 160$ & $117 / 160$ \\
& $=82.5 \%$ & $=93.75 \%$ & $=95.0 \%$ & $=73.12 \%$ \\
\hline Type I Error & $10 / 160$ & $2 / 160$ & $1 / 160$ & $21 / 160$ \\
& $=6.25 \%$ & $=1.25 \%$ & $=0.63 \%$ & $=13.13 \%$ \\
\hline Type II Error & $18 / 160$ & $8 / 160$ & $7 / 160$ & $22 / 160$ \\
& $=11.25 \%$ & $=5.00 \%$ & $=4.37 \%$ & $=13.75 \%$ \\
\hline
\end{tabular}

These tables indicate that CDRs change significantly according to each individual signature. Among these signatures, $(1-4 s) f_{o}$ has the highest CDR, while the other three signatures have lower CDRs. The seventh to ninth rows of Table IV simply sums CDRs and Type I-II errors of training and test data sets. We have then considered four of the signatures together and applied the monolith and partition schemes.

Table V depicts the CDR of LDA for the two schemes under Case 1 together with Type I and Type II error measures. Note that the LDA's correct detection performance improves with the partition scheme. It is also observed that CDRs in both schemes are higher than any of the single signature CDRs given in Tables III and IV.

The bar chart depicted in Fig. 8 presents the CDRs of both single signature processing and multiple signature processing for the two schemes under Case 1. This bar chart affirms that multiple signature processing is more efficient in broken rotor bar fault detection as expressed mathematically in (21)

$$
\begin{aligned}
\mathrm{CDR}_{L D A_{-} \text {partition }}>\mathrm{CDR}_{L D A_{-} \text {monolith }}>\mathrm{CDR}_{(1-4 s) f_{o}} \\
>\mathrm{CDR}_{(1+2 s) f_{o}}>\mathrm{CDR}_{(1-2 s) f_{o}}>\mathrm{CDR}_{(1+4 s) f_{o}} \cdot
\end{aligned}
$$

Similarly, Table VI depicts the CDRs and Type I-II errors of LDA for the two schemes under Case 2. In Case 2, since we have separated the data into training and test sets, we have included the sum of training and test data sets' CDRs, in addition to each set's separate CDR. There is a considerable improvement examined in the CDR with the partition scheme. In addition, these CDRs are higher than any of the single signature's CDRs that are depicted in Tables III and IV with the exception of one equal case.

The partition scheme has provided a way to cope with the nonlinearities in the mapping process and demonstrates an improved correct detection performance with LDA. Partitioning the initial mapping space of the fault detection problem with respect to one of its input variables into smaller disjoint subregions and introducing submapping units, for each of these small subregions, provides an increase in the correct detection performance.

The three-dimensional (3-D) bar chart depicted in Fig. 9 presents the CDRs of both the single signature and multiple 
TABLE IV

CDRs of Single FREQUENCY COMPONENTS UNDER CASE 2

\begin{tabular}{l|c|c|c|c}
\hline & \multicolumn{3}{|c}{ Correct Detection Rate (CDR) } \\
\hline & $(1-2 s) f_{o}$ & $(1+2 s) f_{o}$ & $(1-4 s) f_{o}$ & $(1+4 s) f_{o}$ \\
\hline CDR (Training) & $72 / 80=90.00 \%$ & $76 / 80=95.00 \%$ & $75 / 80=93.75 \%$ & $57 / 80=71.25 \%$ \\
\hline Type I Error (Training) & $4 / 80=5.00 \%$ & $1 / 80=1.25 \%$ & $1 / 80=1.25 \%$ & $10 / 80=12.5 \%$ \\
\hline Type II Error (Training) & $4 / 80=5.00 \%$ & $3 / 80=3.75 \%$ & $4 / 80=5.00 \%$ & $13 / 80=16.25 \%$ \\
\hline CDR (Test) & $61 / 80=76.25 \%$ & $75 / 80=93.75 \%$ & $77 / 80=96.25 \%$ & $61 / 80=76.25 \%$ \\
\hline Type I Error (Test) & $4 / 80=5.00 \%$ & $0 / 80=0.0 \%$ & $0 / 80=0.0 \%$ & $10 / 80=12.5 \%$ \\
\hline Type II Error (Test) & $15 / 80=18.75 \%$ & $5 / 80=6.25 \%$ & $3 / 80=3.75 \%$ & $9 / 80=11.25 \%$ \\
\hline CDR (Training+Test) & $133 / 160=83.13 \%$ & $151 / 160=94.38 \%$ & $152 / 160=95.0 \%$ & $118 / 160=73.75 \%$ \\
\hline Type I Error (Training+Test) & $8 / 160=5.00 \%$ & $1 / 160=0.63 \%$ & $1 / 160=0.63 \%$ & $20 / 160=12.5 \%$ \\
\hline Type II Error (Training+Test) & $19 / 160=11.87 \%$ & $8 / 160=5.00 \%$ & $7 / 160=4.37 \%$ & $22 / 160=13.75 \%$ \\
\hline
\end{tabular}

TABLE V

CDRs With MONOLiTh AND PARTITION SCHEME Under CASE 1

\begin{tabular}{l|c|c}
\hline & \multicolumn{2}{|c}{ Correct Detection Rate (CDR) } \\
\hline & Monolith Scheme & Partition Scheme \\
\hline CDR & $153 / 160=95.63 \%$ & $159 / 160=99.38 \%$ \\
\hline Type I Error & $1 / 160=0.63 \%$ & $0 / 160=0.0 \%$ \\
\hline Type II Error & $6 / 160=3.75 \%$ & $1 / 160=0.63 \%$ \\
\hline
\end{tabular}

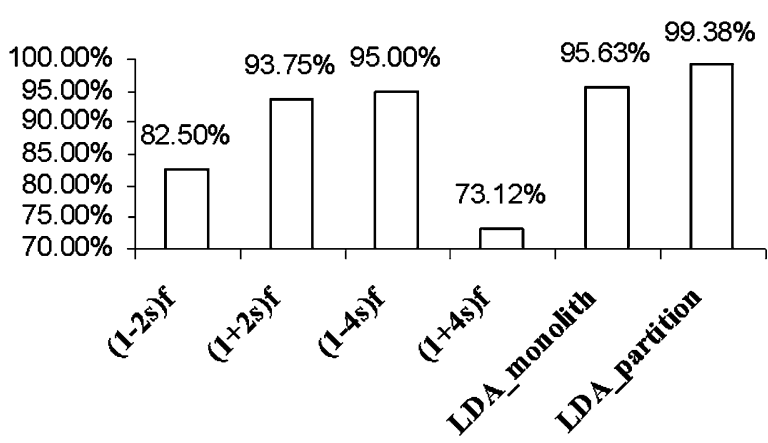

Fig. 8. CDRs with single signature processing and multiple signature processing under Case 1.

TABLE VI

CDRs With Monolith AND PARTITION SCHEMEs UNDER CASE 2

\begin{tabular}{l|c|c}
\hline & \multicolumn{2}{|c}{ Correct Detection Rate (CDR) } \\
\hline CDR (Training) & Monolith Scheme & Partition Scheme \\
\hline Type I Error & $76 / 80=95.00 \%$ & $80 / 80=100.0 \%$ \\
\hline Type II Error & $3 / 80=1.25 \%$ & $0 / 80=0.0 \%$ \\
\hline CDR (Test) & $76 / 80=95.00 \%$ & $78 / 80=97.50 \%$ \\
\hline Type I Error & $0 / 80=0.0 \%$ & $1 / 80=1.25 \%$ \\
\hline Type II Error & $4 / 80=5.0 \%$ & $1 / 80=1.25 \%$ \\
\hline CDR (Total) & $152 / 160=95.00 \%$ & $158 / 160=98.75 \%$ \\
\hline Type I Error & $1 / 160=0.63 \%$ & $1 / 160=0.63 \%$ \\
\hline Type II Error & $7 / 160=4.37 \%$ & $1 / 160=0.63 \%$ \\
\hline
\end{tabular}

signature processing for the two schemes under Case 2. This bar chart clearly indicates that the multiple signature processing is advantageous to the single signature processing and is mathematically expressed in (22). It can be also noticed that CDR is improved with the partition scheme

$$
\begin{aligned}
\mathrm{CDR}_{L D A_{-} \text {partition }}>\mathrm{CDR}_{L D A-\text { monolith }}=\mathrm{CDR}_{(1-4 s) f_{o}} \\
>\mathrm{CDR}_{(1+2 s) f_{o}}>\mathrm{CDR}_{(1-2 s) f_{o}}>\mathrm{CDR}_{(1+4 s) f_{o}} .
\end{aligned}
$$

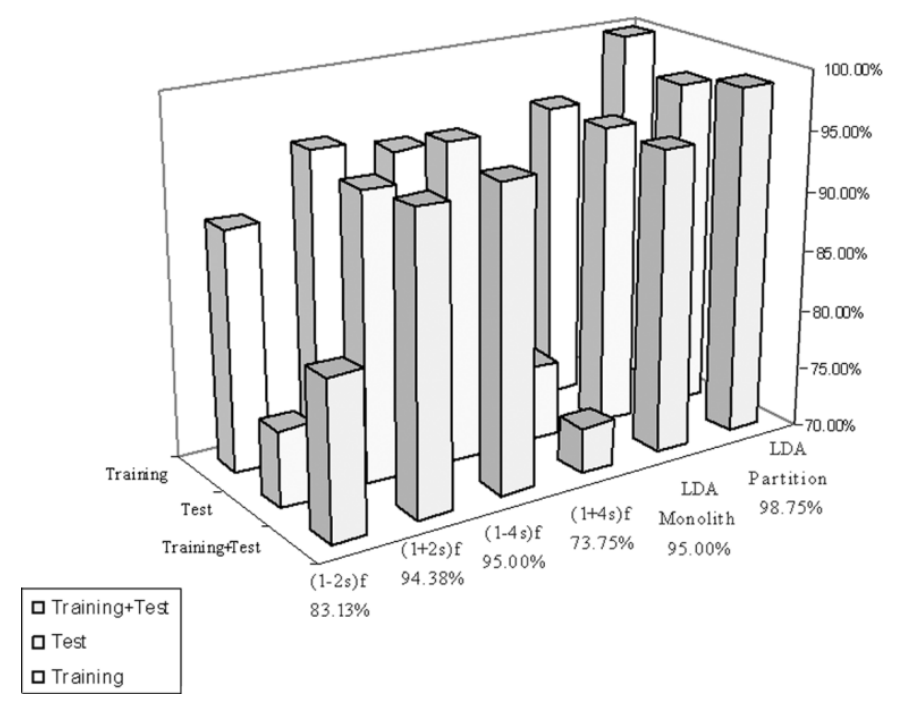

Fig. 9. CDRs with single signature processing and multiple signature processing under Case 2.

In this study, we have investigated two multiple signature processing-based fault detection schemes for a broken rotor bar in induction motors. The investigated fault detection schemes can be adapted to more than one broken rotor bar fault. In addition, the methodology has the appropriate structural formation to be applied to other severe induction motor faults, such as stator winding and bearing faults, where multiple signatures exist within the induction motor that can be utilized in the decision process.

In the monolith and partition schemes, we have applied LDA in the detection units. However, we believe that other methodologies other than LDA can also be applied to the detection units in order to increase the correct detection performance. Among these methodologies, artificial neural networks (ANNs) are perhaps the first methodology that comes to mind. ANN, because of its nonlinear mapping capability, is thought to surmount the nonlinearities in the mapping process and provide an improved detection performance when compared to LDA. Future work will thus investigate the ANN's correct detection performance.

\section{CONCLUSION}

Multiple signature processing for broken rotor bar fault detection is considered to be more reliable and effective than single 
signature processing because of the possibility of the obscuring effects that can overlap the significance of the one and only inspected signature. LDA provides a suitable environment to process multiple signatures for broken rotor bar fault detection. In this paper, we have demonstrated that multiple signature processing provides better accuracy with respect to fault detection performance when compared to single signature processing. In addition to this finding, we have investigated two fault detection schemes for broken rotor bar fault detection with a multiple signature processing feature: the monolith and the partition schemes. Experimental results show that with the partition scheme, correct detection performance of LDA improves. The partition scheme reduces the dimension of the initial mapping space by partitioning it into smaller disjoint subregions and introduces LDA-based submapping units for each particular subregion. This, at the end, provides a better discrimination of broken rotor bar fault from the healthy state of the motor.

\section{REFERENCES}

[1] M. E. H. Benbouzid and G. B. Kliman, "What stator current processing based technique to use for induction motor rotor faults diagnosis?," IEEE Trans. Energy Convers., vol. 18, no. 2, pp. 238-244, Jun. 2003.

[2] B. Li, M.-Y. Chow, Y. Tipsuwan, and J. C. Hung, "Neural-network-based motor rolling bearing fault diagnosis," IEEE Trans. Ind. Electron., vol. 47, no. 5, pp. 1060-1069, Oct. 2000.

[3] S. Altug, M.-Y. Chow, and H. J. Trussell, "Fuzzy inference systems implemented on neural architectures for motor fault detection and diagnosis," IEEE Trans. Ind. Electron., vol. 46, no. 6, pp. 1069-1079, Dec. 1999.

[4] M. Haji and H. A. Toliyat, "Pattern recognition - A technique for induction machines rotor broken bar detection," IEEE Trans. Energy Convers., vol. 16 , no. 4 , pp. 312-317, Dec. 2001.

[5] R. Schoen and T. G. Habetler, "Effects of time varying loads on rotor fault detection in induction machines," IEEE Trans. Ind. Appl., vol. 31, no. 4, pp. 900-906, Jul./Aug. 1995.

[6] G. B. Kliman et al., "Non-invasive detection of broken rotor bars in operating induction motors," IEEE Trans. Energy Convers., vol. EC-3, no. 4, pp. 873-879, Dec. 1988.

[7] F. Filipetti et al., "AI techniques in induction machines diagnosis including the speed rifle effect," in Proc. IEEE Industry Applications Society Annu. Meet. Conf., San Diego, CA, Oct. 6-10, 1996, pp. 655-662.

[8] S. L. Marple, Digital Spectral Analysis with Applications. Upper Saddle River, NJ: Prentice-Hall, 1987.
[9] L. Lebart, A. Morineau, and K. M. Warwick, Multivariate Descriptive Statistical Analysis. New York: Wiley, 1984.

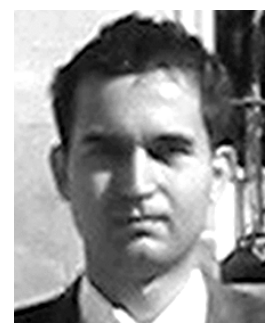

Bulent Ayhan (S'01) received the B.S. and M.S. degrees in electrical and electronics engineering from Bogazici University, Istanbul, Turkey, in 1998 and 2000 , respectively. He is currently pursuing the Ph.D. degree as a Research Assistant in Advanced Diagnosis Automation and Control Lab (ADAC) at North Carolina State University, Raleigh.

His research interests include intelligent health monitoring of rotating machinery systems.

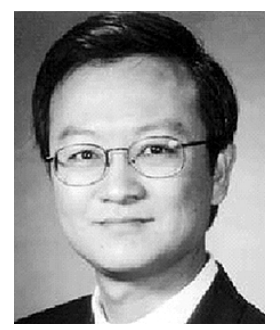

Mo-Yuen Chow (S'81-M'82-SM'93) received the B.S. degree in electrical and computer engineering from the University of Wisconsin, Madison, in 1982, and the M.Eng. and Ph.D. degrees from Cornell University, Ithaca, NY, in 1983 and 1987, respectively.

Currently, he is Professor in the Department of Electrical and Computer Engineering, North Carolina State University (NCSU), Raleigh. His core technology is diagnosis and control, artificial neural network (ANN) and fuzzy logic with applications to areas including motors, process control, power systems, and communication systems. He has established the Advanced Diagnosis Automation and Control (ADAC) Laboratory at NCSU.

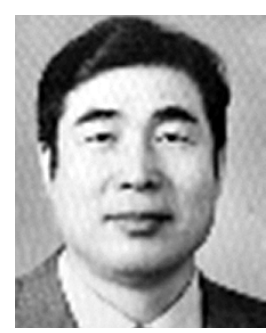

Myung-Hyun Song (M’95) was born in Jeju, Korea, in 1953. He received the B.S., M.S., and Ph.D. degrees in electrical engineering from Korea University, Seoul.

Currently, he is Professor of the School of Information and Communication, Sunchon National University, Sunchon, Chonnam, Korea, where he has been since 1988. From 1977 to 1981, he was with Samsung Electronic Co., Suwon, Korea, involved with the design and application of electric motors. His current research interests include induction motor fault detection and diagnosis, factory automation, and web-based control. 